\title{
Theoretical Heights and Durations of Echoes From Large Meteors'
}

\author{
Laurence A. Manning \\ Contribution From the Radioscience Laboratory, Stanford University, Stanford, Calif.
}

(Received March 19, 1964)

\begin{abstract}
Recent studies have shown that attachment as well as diffusion is important in determining the electron-density distribution about the path of a meteor. The present paper develops in detail the mathematical theory of the durations of radio echoes from overdense trails. Included is the dependence of the height distribution of ionization on meteor magnitude, velocity, zenith angle, the form of the attachment law, the height dependence of diffusion coefficient, and an adjustable relation between luminous and ionizing efficiency. It is shown that well defined attachment-free and attachment-controlled duration regions exist with different line-density and wavelength dependences. The transition zone is broad, and its location depends strongly on meteor velocity. Normalized duration and line-density parameters are defined in terms of which a single computer-calculated duration versus density relation good for all parameter values is plotted. Bridging formulas approximating the duration relation are derived from asymptotic expressions, and the relation between echoing height and duration or line density is presented. Equations are given relating the exponent of the wavelength to echo duration. In a companion paper, the theory will be applied to experimental data and the values of the physical parameters and constants will be derived.
\end{abstract}

\section{Introduction}

When the electron line density created by the passage of a meteor is appreciably less than $10^{14}$ electrons/meter, the reflected amplitude decays exponentially with a time constant determined by the wavelength and height-dependent diffusion coefficient. Such trails are called underdense, and an incident wave penetrates the trail with little change in amplitude or phase. When, however, the line density appreciably exceeds $10^{14}$ electrons/meter, an incident wave is significantly affected in phase and amplitude by the ionization distribution. Such an "overdense" trail will return a strong echo primarily during the period of time when a negative dielectric constant core exists about the trail axis (underdense echoes may also have overdense cores, but only of radius small compared with the skin depth, thus not controlling the echo duration). If the electrondensity distribution of an overdense trail is controlled entirely by diffusion, the duration of the echo reflected perpendicularly from a given height on a smooth cylindrical trail is directly proportional to the line density [Manning and Eshleman, 1959]. However, the duration of overdense echoes does not increase as rapidly as predicted by this relation for

1 Jointly supported by the U.S. Army Signal Corps, the U.S. Air Force, the U.S. Navy (Office of Naval Research) and by the National Science Foundation, Grant NSF-GP948. very large meteors. Davis, Greenhow, and Hall [1959 ] have suggested that a two-body attachment process is important in removing electrons, thus shortening the durations. Greenhow and Hall [1962] have more recently suggested that a three-body attachment process may better fit the experimental results. These authors, however, have not presented a general theory for echo duration including the effect of attachment, from which a careful study of the influence of the relevant parameters could be made.

In the present paper a general analytic theory of overdense meteor-echo duration will be given for two-, three-, or m-body attachment, including the effect of variation of diffusion coefficient with height, ionization production with velocity, the wavelength, and other pertinent parameters. In computing duration, it will be assumed that an echo will be received if an overdense core exists at any point along the trail. With attachment included, it will be shown that meteor echoes fall into three groups each of which has a characteristic duration behavior: (1) underdense, (2) overdense without electron loss, and (3) overdense with attachment. By suitable normalization, simple formulas are found for each region; a simple bridging formula connects the latter two domains in a region of great interest. In a following paper, the theory will be compared with a variety of experimental results, and values deduced for the physical parameters. 


\section{Reflection Conditions and Duration}

At a given point on the ionization trail let the electron line density be $q$ electrons/meter. Then in the notation of Manning and Eshleman [1959], a useful normalized line density is

$$
Q=\pi r_{e} q=0.885 \times 10^{-14} q
$$

where $r_{e}$ is the electron radius. If $Q<<1$, the trail is underdense, and except at very high frequencies or in the presence of transverse-polarization plasma resonance [Manning and Eshleman, 1959], the reflection coefficient is

$$
\rho=Q \exp \left(-\frac{t}{T}\right) \quad \text { for } Q<1
$$

where by definition $\rho=\left(2 \pi^{2} R / \lambda\right)^{\frac{1}{2}} \cdot\left(E_{\text {sc }} / E_{\text {inc }}\right)$, with $R$ the radar range, $\lambda$ the wavelength, $E_{\mathrm{se}}$ the received electric field strength, and $E_{\mathrm{inc}}$ the incident field strength at the trail; in (1) $T$ is the underdense exponential-decay time constant, given by

$$
T=\frac{\lambda^{2}}{16 \pi^{2} D}
$$

with $D$ the ambipolar diffusion coefficient, and $\lambda$ the wavelength.

For overdense trails the echo duration can be equated to the duration of a negative-dielectricconstant core in a diffusion-formed gaussian radial ionization distribution. The radius of the core is given by $r^{2}=4 D t \ln (4 Q T / \pi t)$, where $t$ is the time after trail formation [Manning and Eshleman, 1959]. This radius shrinks to zero at a given point on the trail when $4 Q T / \pi t=1$, so that the high-density trail duration $t_{H}$ is

$$
t_{H}=\frac{4 Q T}{\pi}
$$

Equation (4) gives the duration attainable from a particular portion of an overdense trail. Note that $Q$ and $T$ are both functions of height. In the presence of attachment of trail electrons to neutral particles according to a relation of the form $d N / d t \propto$ $N$, where $N$ is electron volume density, $Q$ must be considered a function of time as well as height. Since the rate of loss of electrons by attachment is proportional to the existing volume density, all volume elements lose density in proportion and the gaussian diffusion distribution is not disturbed; attachment can thus be considered to cause an exponential line-density decrease with time.

\section{Line-Density Distribution and Trail Height}

In the absence of flares and fragmentation, the distribution of line density with height can be de- scribed by Herlofson's formula

$$
Q=\frac{9}{4} Q_{m_{o}}\left(1-\frac{u}{3}\right)^{2} u \quad u \leq 3
$$

where $Q_{m o}$ is the maximum line density, and

$$
u=\exp \left(-\frac{h-h_{m o}}{H}\right)
$$

with $h$ the height, $h_{m o}$ the height of maximum line density $Q_{m o}$, and $H$ the atmospheric scale height. In the presence of attachment, $Q$ will be less than that given by (5) by a decreasing exponential time factor whose time constant depends on height. The form of height dependence depends on the attachment mechanism, being proportional to atmospheric density for a two-body process, and proportional to density squared for a three-body process. In general, the attachment time constant is

$$
A \cdot \exp \left[\frac{m(h-95)}{H}\right]
$$

if $A$ is the time constant at an arbitrary reference height of $95 \mathrm{~km}, h$ is height, $H$ is scale height, and $m$ is zero for height-independent attachment, $m=1$ for two-body attachment, and $m=2$ for three-body attachment.

Including attachment, the line-density (5) may now be written

$$
Q=\frac{9}{4} Q_{m_{o}}\left(1-\frac{u}{3}\right)^{2} u \exp \left\{-\frac{t}{A} \exp \left[-\frac{m(h-95)}{H}\right]\right\}
$$

with $u \leq 3$. In (7), the term $\exp [-m(h-95) / H]$ is equal to $u^{m} \cdot \exp \left[m\left(95-h_{m o}\right) / H\right]$. Drawing on the physical theory of meteors, the latter exponential is dependent on $Q_{m_{0}}$.

The pertinent aspects of the physical theory of meteors are conveniently summarized by McKinley [1961]. Eliminating mass from his equations (7-7) and (7-17) yields for the atmospheric density at maximum ionization

$$
\rho \propto \frac{q_{\max }^{1 / 3} \cos ^{2 / 3} Z}{\tau_{q}^{1 / 3} V^{8 / 3}}
$$

where $q_{\max }$ is $Q_{m o} / \pi r_{e}, Z$ is the zenith angle of the meteoric radiant, $V$ is the meteor velocity, and $\tau_{q}$ is the ionization-efficiency factor. Equation (8) differs from McKinley's equation (7-22) only in the retention of the zenith angle $Z$. Comparing McKinley's equations (7-4) and (7-5), the ionization $q$ is related to the luminosity $I$ by

$$
q=\frac{\tau_{q}}{\tau_{I}} \frac{I}{\eta V}
$$


where $\tau_{I}$ is the luminous-efficiency factor, and $\eta$ is the mean ionization potential of the atoms involved. We shall assume that the ratio of line density to luminosity is proportional to $V^{J}$. Then from (9), $\tau_{q} / \tau_{\tau} \propto V^{J+1}$; using Whipple's simplification of Opik's theoretical calculations as given in (7-5) of McKinley, $\tau_{I} \propto V$. Hence we assume $\tau_{q}$ proportional to $V^{J+2}$, and (8) may be written

$$
\rho \propto \frac{Q_{m o}^{1 / 3} \cos ^{2 / 3} Z}{V^{(10+J) / 3}} .
$$

From analysis of the radio and photographic data, Millman and McKinley [1956] and Whipple [1955] independently concluded $J=1$. However, when attachment is considered the most suitable value of $J$ depends on the attachment law assumed, so it is appropriate to leave $J$ to be determined again by reference to the experimental results. The assumption that $J \neq 0$ implies that a relation exists between line density and visual magnitude of the form $Q_{m o}=Q_{0}\left(V / V_{0}\right)^{J} 10^{-M / 2.5}$.

For our purposes (10) can be written more conveniently in the form

$$
\exp \left(\frac{95-h_{m o}}{H}\right)=K \frac{Q_{m o}^{1 / 3}}{v^{(10+) J / 3}}
$$

by assuming an exponential atmosphere; the constant $K$ depends on the atmospheric density at the arbitrary reference height of $95 \mathrm{~km}$, and in a complicated way, on the ionization potential, heat of ablation, heat transfer coefficient, meteoric density, scale height, shape factor, etc. The velocity and zenith-angle variations have been normalized using the relation

$$
v=\left(\frac{V}{V_{0}}\right)(\sec Z)^{2 /(10+J)}
$$

so $v=1$ for a $V_{0} \mathrm{~km} / \mathrm{s}$ meteor in the zenith.

Using (11), the line-density distribution equation (7) can be written

$$
Q=Q_{m o} \cdot \frac{9}{4}\left(1-\frac{u}{3}\right)^{2} u \exp \left(-B u^{m}\right) \quad u \leq 3
$$

where $B$ is defined by

$$
B=\frac{t}{A}\left(K \frac{Q_{m o}^{1 / 3}}{v^{(10+J) / 3}}\right)^{m} .
$$

\section{Derivation of Duration}

From (4) the duration at height $h$ (or $u$ ) is $t_{H}=$ $4 Q T / \pi$. By (3), $T=\lambda^{2} /\left(16 \pi^{2} D\right)$; the diffusion coefficient $D$ varies with height as $D=D_{0} \exp [(h-95) /$ $H]$ if $D_{0}$ is the value at $95 \mathrm{~km}$ (about $4 \mathrm{~m}^{2} / \mathrm{s}$ ). Using (11), the time constant $T_{m o}$ at height of maximum onization is then

$$
T_{m o}=\frac{\lambda^{2}}{16 \pi^{2} D_{0}} \frac{K Q_{m o}^{1 / 3}}{v^{(10+J) / 3}} .
$$

The time constant at height $h$ is $T_{m o} \exp \left[-\left(h-h_{m o}\right) /\right.$ $H] \equiv T_{m o} u$, hence the exponential-decay time constant $t$ at any height is

$$
T=u \cdot \frac{\lambda^{2}}{16 \pi^{2} D_{0}} \frac{K Q_{m o}^{1 / 3}}{v^{(10+J) / 3}} .
$$

Combining (13) and (15), the duration relation $t_{H}=4 Q T / \pi$ for a particular height described by $u$ becomes

$$
t_{H}=\frac{9 \lambda^{2} K Q_{m o}^{4 / 3}}{16 \pi^{3} D_{0} v^{(10+J) / 3}}\left[\left(1-\frac{u}{3}\right)^{2} u^{2} \exp \left(-B u^{m}\right)\right] \quad u \leq 3 .
$$

To find the actual echo duration, we must evaluate (16) at the value of $u$ (height) equal to $u_{m}$ for which the duration is the greatest. To do so, define

$$
y=\left(1-\frac{u}{3}\right)^{2} u^{2} \exp \left(-B u^{m}\right) .
$$

The duration is maximum when $d y / d u=0$, a condition that requires

$$
B_{H}=\frac{2\left(1-2 u_{m} / 3\right)}{m u_{m}^{m}\left(1-u_{m} / 3\right)} .
$$

Values of $u_{m}$ must lie in the limits $0 \leq u_{m} \leq 3 / 2$; the subscript $H$ on $B$ implies that $B_{H}$ is evaluated with $t=t_{H}$, the maximum duration. It may be noted that if $m=0$ (attachment independent of height) $u_{m}=3 / 2$, just as in the case of no attachment. The height of maximum duration is then below the height of maximum electron production by $\Delta h=H \cdot \ln$ $3 / 2=0.40547 H \sim 2.4 \mathrm{~km}$. Figure 1 (a) shows how $B_{H}$ (proportional to duration) varies with $u_{m}=$ $\exp \left[-\left(h_{m}-h_{m_{0}}\right) / H\right]$, and figure $1 \mathrm{~b}$ shows how $B_{H}$ varies with the relative height $\left(h_{m}-h_{m o}\right) / H$, where $h_{m}$ is the height of maximum duration.

Combining (14), (16), (17), and (18), two relations are obtained in duration $t_{H}$, line density $Q_{m o}$, and $u_{m}$ :

$$
\begin{aligned}
& t_{H} \cdot \frac{16 \pi^{3} D_{0} v^{(10+J) / 3}}{9 \lambda^{2} K Q_{m o}^{4 / 3}}=y\left(u_{m}\right) \\
& =\left(1-\frac{u_{m}}{3}\right)^{2} \exp \left[-\left(\frac{2}{m}\right) \frac{\left(1-2 u_{m} / 3\right)}{\left(1-u_{m} / 3\right)}\right] u_{m}^{2}
\end{aligned}
$$

and

$$
t_{H} \cdot \frac{1}{A}\left[\frac{K Q_{m o}^{1 / 3}}{v^{(10+J) / 3}}\right]^{m}=B_{H}=\frac{2}{m} \frac{1}{u_{m}^{m}} \frac{1-2 u_{m} / 3}{1-u_{m} / 3} .
$$

Equations (19) and (20) are indeterminate if $m=0$. If $m \neq 0, t_{H}$ can in principle be found as a function of $Q_{m_{0}}$ by eliminating $u_{m}$ between (19) and (20). This elimination can be performed in the asymptotic limits of low and high attachment. However, exact calculation of the relation between $t_{H}$ and 


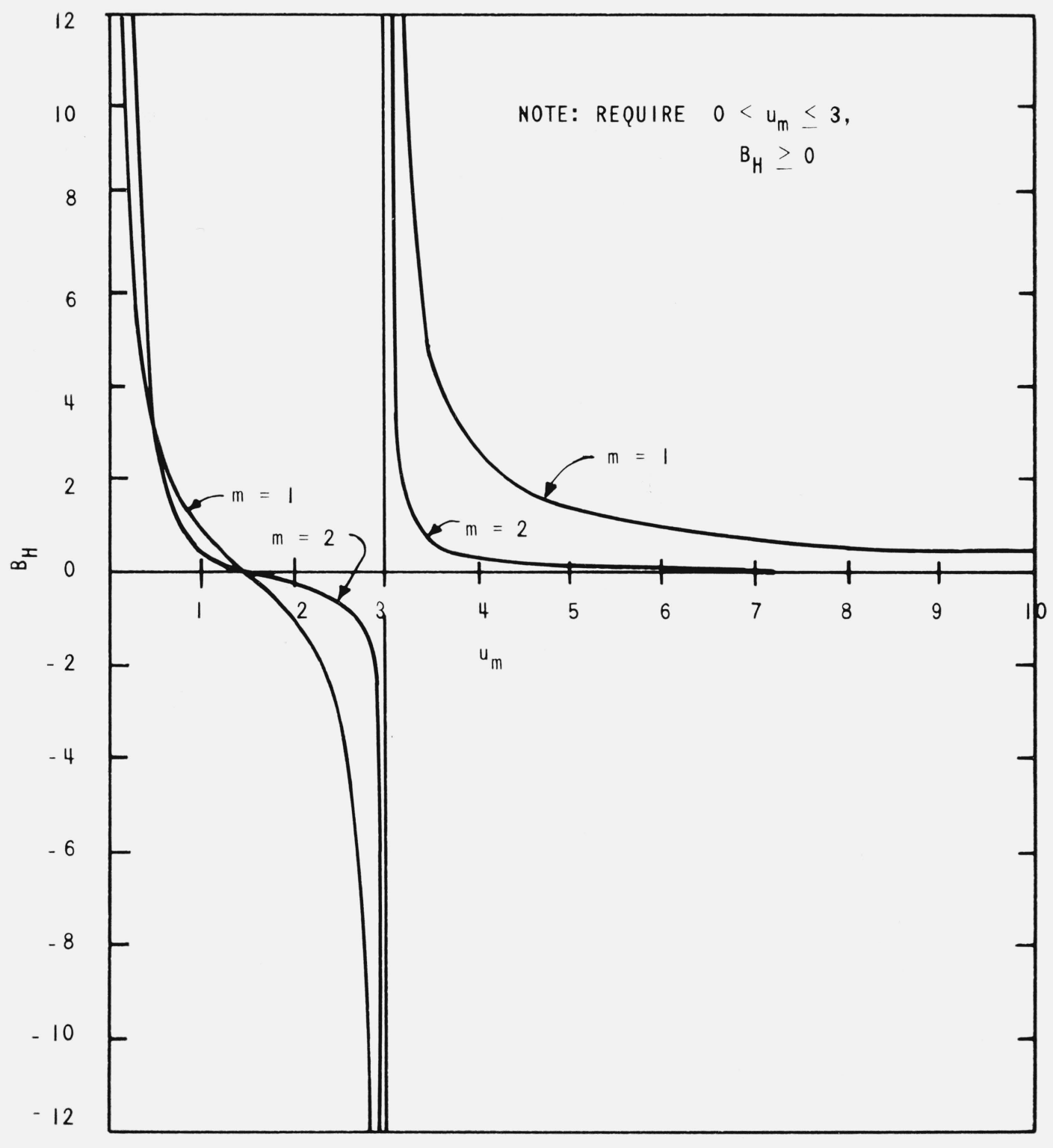

FIGURE 1. Variations of $\mathrm{B}_{\mathrm{H}}$ with height parameters.

(a) Variation of $B_{H}$, as given by (18), with $u_{m}=\exp \left[-\left(h_{m}-h_{\mathrm{mo}}\right) / H\right]$

$Q_{m o}$ must be carried out by treating $u_{m}$ as a parameter and finding a corresponding $t_{H}$ and $Q_{m o}$ for each $u_{m}$.

\section{Normalized Duration and Line-Density Variables}

To avoid having to calculate families of duration curves for different velocities, wavelengths, etc., (19) and (20) will be put in a normalized form. Let $z$ be normalized duration $t_{H}$, and $x$ normalized maximum line density $Q_{m o}$. Then (19) and (20) can be written

$$
\frac{z}{x^{4 / 3}}=y \quad z \cdot x^{m / 3}=B_{H}
$$

Solving for $z$ and $x$,

$$
\begin{gathered}
z=B_{H}^{4 /(4+m)} \cdot y^{m /(4+m)} \\
x=\left(\frac{B_{H}}{y}\right)^{3 /(4+m)} .
\end{gathered}
$$

Putting in $y$ and $B_{H}$ from the left-hand side of (19) and $(20)$, 


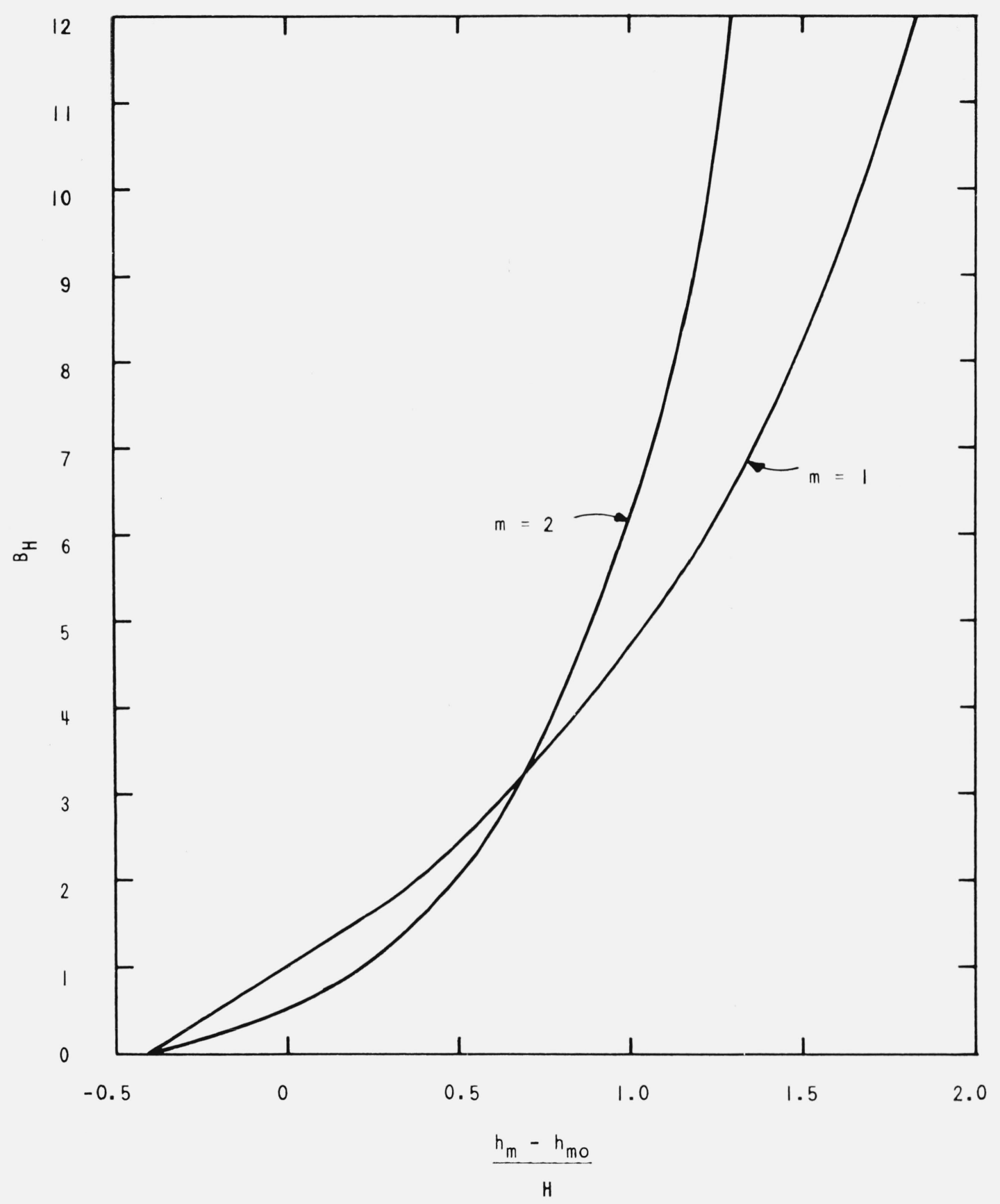

Figure 1. Variations of $\mathrm{B}_{\mathrm{H}}$ with height parameters.-Continued (b) Plot of $B_{H}$ versus $\left(h_{m}-h_{\mathrm{mo}}\right) / H$

$$
z=t_{H} \frac{K^{3 m /(4+m)} D_{0}^{m /(4+m)}\left(\frac{4 \pi^{3 / 2}}{3}\right)^{2 m /(4+m)}}{A^{4 /(4+m)} \cdot v^{m(10+J) /(4+m)} \cdot \lambda^{2 m /(4+m)}}
$$

and

$$
x=Q_{m o} \frac{K^{3(m+1) /(4+m)} \cdot \lambda^{6 /(4+m)}}{A^{3 /(4+m)} v^{(10+J)(m+1) /(4+m)} D_{0}^{3 /(4+m)}\left(\frac{4 \pi^{3 / 2}}{3}\right)^{6 /(4+m)}} .
$$

The cases $m=0,1$, and 2 are of especial interest For height-independent attachment, $m=0$,

$$
\begin{gathered}
z=\frac{t_{H}}{A} \\
x=Q_{m o} \frac{K^{3 / 4} \lambda^{3 / 2}}{A^{3 / 4} v^{(10+J) / 4} D_{0}^{3 / 4}\left(\frac{4 \pi^{3 / 2}}{3}\right)^{3 / 2}} .
\end{gathered}
$$


For two-body attachment, $m=1$,

$$
\begin{gathered}
z=t_{H} \frac{K^{3 / 5} D_{0}^{1 / 5}\left(\frac{4 \pi^{3 / 2}}{3}\right)^{2 / 5}}{A^{4 / 5} v^{(10+J) / 5} \lambda^{2 / 5}} . \\
x=Q_{m o} \frac{K^{6 / 5} \lambda^{6 / 5}}{A^{3 / 5} v^{2(10+J) / 5} D_{0}^{3 / 5}\left(\frac{4 \pi^{3 / 2}}{3}\right)^{6 / 5}} .
\end{gathered}
$$

For three-body attachment, $m=2$,

$$
\begin{gathered}
z=t_{H} \frac{K D_{0}^{1 / 3}\left(\frac{4 \pi^{3 / 2}}{3}\right)^{2 / 3}}{A^{2 / 3} v^{(10+J) / 3} \lambda^{2 / 3}} \\
x=Q_{m_{0}} \frac{K^{3 / 2} \lambda}{A^{1 / 2} v^{(10+J) / 2} D^{1 / 2}\left(\frac{4 \pi^{3 / 2}}{3}\right)} .
\end{gathered}
$$

It may be noted that $4 \pi^{3 / 2} / 3=7.4242$.

\section{Duration Versus Line Density}

Exact curves of normalized duration $z$ versus normalized line density $x$ have been calculated for $m=0,1$, and 2 using a digital computer. For the simple case of $m=0$, (16) reduces, upon substituting $z$ and $x$ from $(26)$, to the explicit relation

$$
z e^{z}=\left(\frac{9}{16}\right) x^{4 / 3} \quad \text { for } m=0 .
$$

However, for $m=1$ and $2, y$ and $B$ were computed versus $u_{m}$ from (19) and (20), and $z$ and $x$ were computed from $y$ and $B$ using (22) and (23). The results are shown in figure 2 , and tabulated in table 1 for key values with $m=1$ and 2 . The meaning of the coordinates $x$ and $z$ is defined by (26), (27), and (28) for $m=0,1$, and 2 ; it is different for each of the curves.

In examining figure 2 , note that $\log x$, the abscissa, is a linear function of the meteor magnitude. Note also that when $x$ and $z$ are much less than one, attachment is not important, and duration is proportional to $Q_{m o}^{4 / 3}$. When $x$ and $z$ are much greater than one, the duration for two-body attachment $(m=1)$ approaches proportionality to $Q_{m o}^{2 / 9}$. For three-body attachment $(m=2)$ the proportion is to $Q_{m o}^{1 / 3}$. If $m=0$, corresponding to height-independent attachment, a logarithmic relation is approached, but this case does not correspond well to the physical situation. The transition region is of especial interest, since it covers a line-density range of about 100 to 1 (5 magnitudes). In figure 3 the transition is plotted to a larger scale. No appreciable difference between the normalized curves for $m=1$ and $m=2$ occurs until $x$ is greater than about

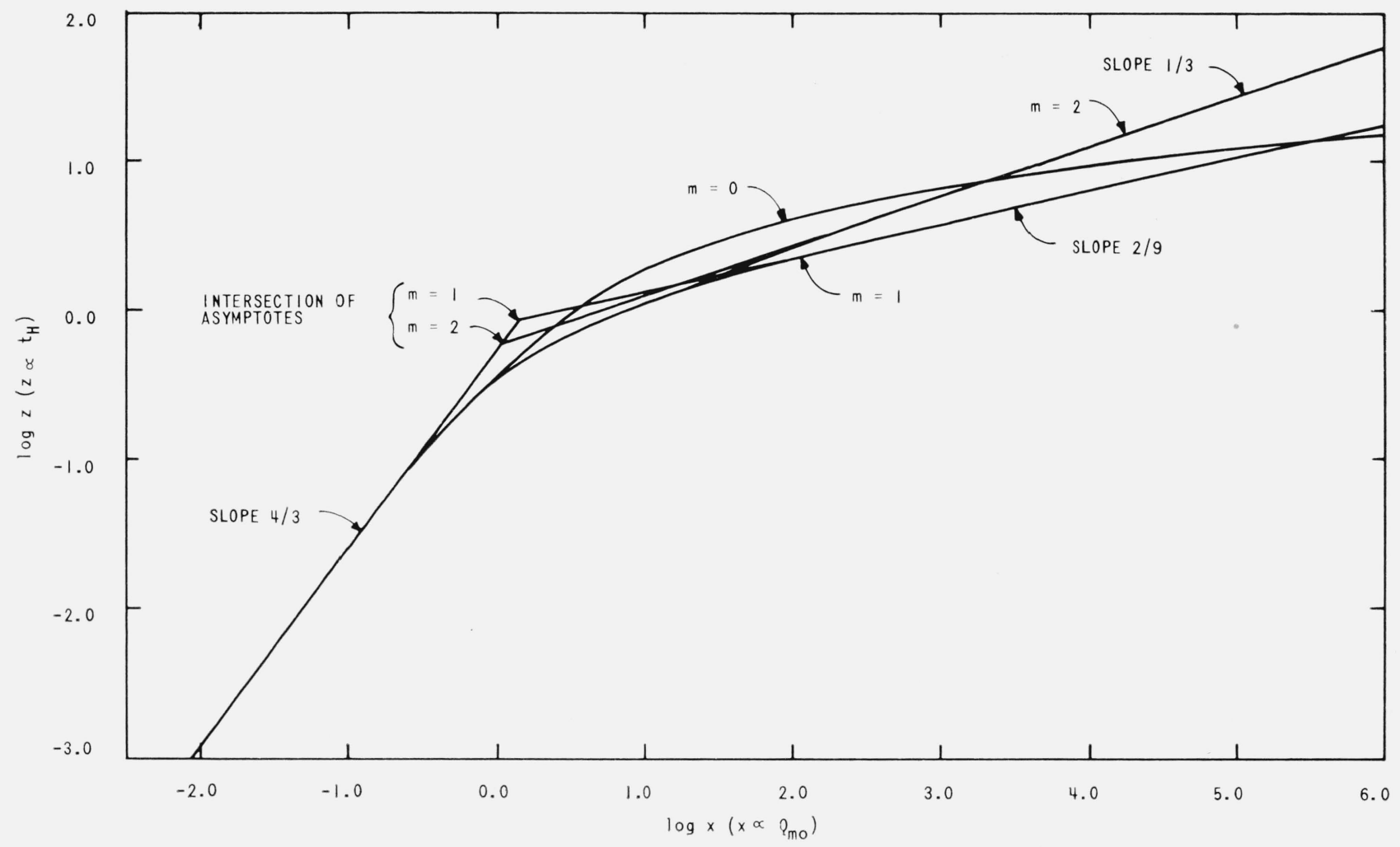

FIGURE 2. The variation of normalized echo duration z versus normalized line density $\mathrm{x}$.

Height-independent attachment corresponds to $m=0$; two- and three-body attachment corresponds to $m=2$ and 3 . 
1.5 , but the variables $x$ and $z$ have different meanings in the two cases. In a group of sporadic meteors transition behavior will be noted over an even greater magnitude range, because of

TABLE 1. Normalized duration and line density

\begin{tabular}{|c|c|c|c|c|c|c|}
\hline \multirow{3}{*}{$u_{m}$} & \multicolumn{3}{|c|}{$m=1$} & \multicolumn{3}{|c|}{$m=2$} \\
\hline & \multirow{2}{*}{$\log x$} & \multirow{2}{*}{$\log z$} & \multirow{2}{*}{$\frac{d(\log z)}{d(\log y)}$} & \multirow{2}{*}{$\log x$} & \multirow{2}{*}{$\log z$} & $d(\log z)$ \\
\hline & & & & & & $d(\log y)$ \\
\hline 1. 498 & -1.318 & -2.009 & 1.324 & -1.337 & -2.034 & 1.326 \\
\hline 1. 495 & -1.077 & -1.692 & 1. 311 & -1.137 & -1.768 & $\begin{array}{l}1.320 \\
1.320\end{array}$ \\
\hline 1. 480 & -0.7056 & -1.213 & 1.250 & -0.8294 & -1.367 & 1. 282 \\
\hline $\begin{array}{l}1.450 \\
\text { 1. }\end{array}$ & $\begin{array}{l}-.4462 \\
\end{array}$ & -0.9019 & 1. 140 & -.6171 & -1.101 & 1.210 \\
\hline $\begin{array}{l}1.40 \\
1.40\end{array}$ & -.2315 & -.8710 & 0.9997 & -.4436 & -0.8995 & 1.110 \\
\hline 1. 20 & .1917 & -.3191 & .6673 & -.1026 & -.5670 & 0.8342 \\
\hline 1.0 & .4718 & -.1572 & .4999 & .1341 & -.3904 & .6660 \\
\hline 0.8 & .7305 & -.04187 & .3997 & .3685 & -.2481 & .5547 \\
\hline .6 & 1.012 & .06056 & .3335 & .6409 & -.1085 & .4761 \\
\hline .4 & 1. 368 & .1701 & .2856 & 1.005 & -.0530 & .4162 \\
\hline .2 & 1. 939 & .3213 & .2500 & 1.613 & .2901 & .3702 \\
\hline .1 & 2. 492 & .4550 & .2353 & 2. 216 & .5069 & .3508 \\
\hline .06 & 2. 895 & .5487 & .2305 & 2. 660 & .6609 & .3439 \\
\hline .04 & 3. 214 & .6215 & .2267 & 3. 012 & .7813 & .3396 \\
\hline .03 & 3. 440 & .6727 & .2259 & $\begin{array}{l}\text { 3. } 262 \\
\text { 3. }\end{array}$ & .8660 & .3384 \\
\hline .02 & $\begin{array}{l}3.758 \\
3.750\end{array}$ & .7443 & .2246 & $\begin{array}{l}\text { 3. } 615 \\
\text { 3. }\end{array}$ & .9849 & $\begin{array}{l}.0007 \\
.3366\end{array}$ \\
\hline .01 & 4. 300 & .8659 & .2234 & 4.217 & 1.187 & .3350 \\
\hline
\end{tabular}

the strong dependence of $x$ and $z$ on velocity. For instance if $J=1$, for two-body attachment a 3 to 1 spread in velocity will cause a $v^{(10+J) / 5}$ or an 11 to 1 shift in $z$ and a 125 to 1 shift in $x$. For threebody attachment, the shifts are 56 to 1 in $z$ and 425 to 1 in $x$. On a plot of $\log t_{\mathrm{H}}$ versus $\log Q_{\mathrm{mo}}$, an increase in velocity slides the curve toward higher coordinate values in a direction with slope $m /(m+1)$ as sketched in figure 4 for $m=1$ and $m=2$. The length of the vector in the plots is drawn for a velocity shift of 2 to 1 . Similarly, a change in wavelength causes the duration curve to shift toward the second quadrant with slope $-m / 3$. The wavelength vectors in figure 4 are again drawn for a 2 to 1 increase in $\lambda$. Increases in attachment time constant $A$ shift the curve up to the right with slope $4 / 3$, and increases in diffusion coefficient shift the curve down to the right with slope $-m / 3$.

The variation of $\log x$ with $\log u_{m}$ is plotted in figure 5. This relation will be of especial use in the data analysis, since $\log x=\log Q_{\text {mo }}+$ constant, and varies linearly with the meteoric magnitude $M$. On the other hand $\log u_{m}=\log \exp \left[-\left(h_{m}-h_{\mathrm{mo}}\right) /\right.$ $H]=-0.434294\left(h_{m}-h_{\mathrm{mo}}\right) / H$, and is proportional to the difference of the final echoing height and the height of maximum ionization.

\section{Behavior of Duration in Asymptotic Regions}

Inspection of figure 2 shows that when $x$ and $z$ are much less than one, attachment may be neglected. Equation (29) then reduces to

$$
z=\frac{9}{16} x^{4 / 3} \quad x<<1, z<<1
$$

for $m=0$, and identical relations are found for other $m$ 's by placing $B=0$ so that $u_{m}=3 / 2$, and putting the resulting $y$ of (19) in the first of (21). In this attachment-free region, if $z$ and $x$ are given their values from (24) and (25),

$$
t_{H}=Q_{m o}^{4 / 3} \frac{K \lambda^{2}}{v^{(10+J) / 3} D_{0}\left(\frac{4 \pi^{3 / 2}}{3}\right)^{4}} \quad x, z<<1 .
$$

When $x$ and $z$ are much greater than one, equally simple equations exist. For $m=0$, the general equation (29) can be used without approximation. For other values of $m$, as $x$ and $z$ become large, (21) shows $B$ is large too, and (20) shows that $u_{m}$ is small. Then using (19) and (20)

$$
B \cong \frac{2}{m} \frac{1}{u_{m}^{m}} \text { and } y \cong u_{m}^{2} e^{-2 / m}
$$

and from (21)

$$
z \cong x^{2 m / 3(m+2)}(2 / m)^{2 /(m+2)} e^{-2 /(m+2)} \quad m>0 .
$$

In particular, if $m=1$ (two-body attachment)

$$
z \simeq x^{2 / 9} \frac{2^{2 / 3}}{e^{2 / 3}} \quad x, z>>1
$$

and

$$
t_{H} \cong Q_{m o}^{2 / 9} \frac{A^{2 / 3} v^{(10+J) / 9} \lambda^{2 / 3} 2^{3 / 3}}{K^{1 / 3} D_{0}^{1 / 3}\left(\frac{4 \pi^{3 / 2}}{3}\right) e^{2 / 3}}
$$

if $m=2$ (three-body attachment)

$$
z \simeq \frac{x^{1 / 3}}{e^{1 / 2}} \quad x, z>>1
$$

and

$$
t_{H} \cong Q_{m o}^{1 / 3} \frac{A^{1 / 2} v^{(10+J) / 6} \lambda}{K^{1 / 2} D_{0}^{1 / 2}} \frac{3}{4 \pi^{3 / 2} e^{1 / 2}} .
$$

Also of interest are the values of $x$ and $z$ at which the asymptotic relations (33) and (35) intersect (30). When $m=1$, the intersection occurs for $x=1.3971$, $\log x=0.14493$, and for $z=0.87772, \log z=-0.05664$. When $m=2$, the intersection occurs for $x=1.0783$, $\log x=0.03273$, and for $z=0.62196, \log z=-0.20624$.

\section{Bridging Formulas}

When $m=0, x^{4 / 3}=(16 / 9) z \cdot \exp (z)$ is exact. For $m \neq 0$, an exact and explicit relation between $x$ and $z$ does not exist. However, by taking the harmonic mean of (30) with (33) or (35), sufficiently accurate, explicit bridging relations can be derived that are useful for all values of $x$ and $z$. When $m=1$, to within about 6 percent in the transition zone and better elsewhere, 


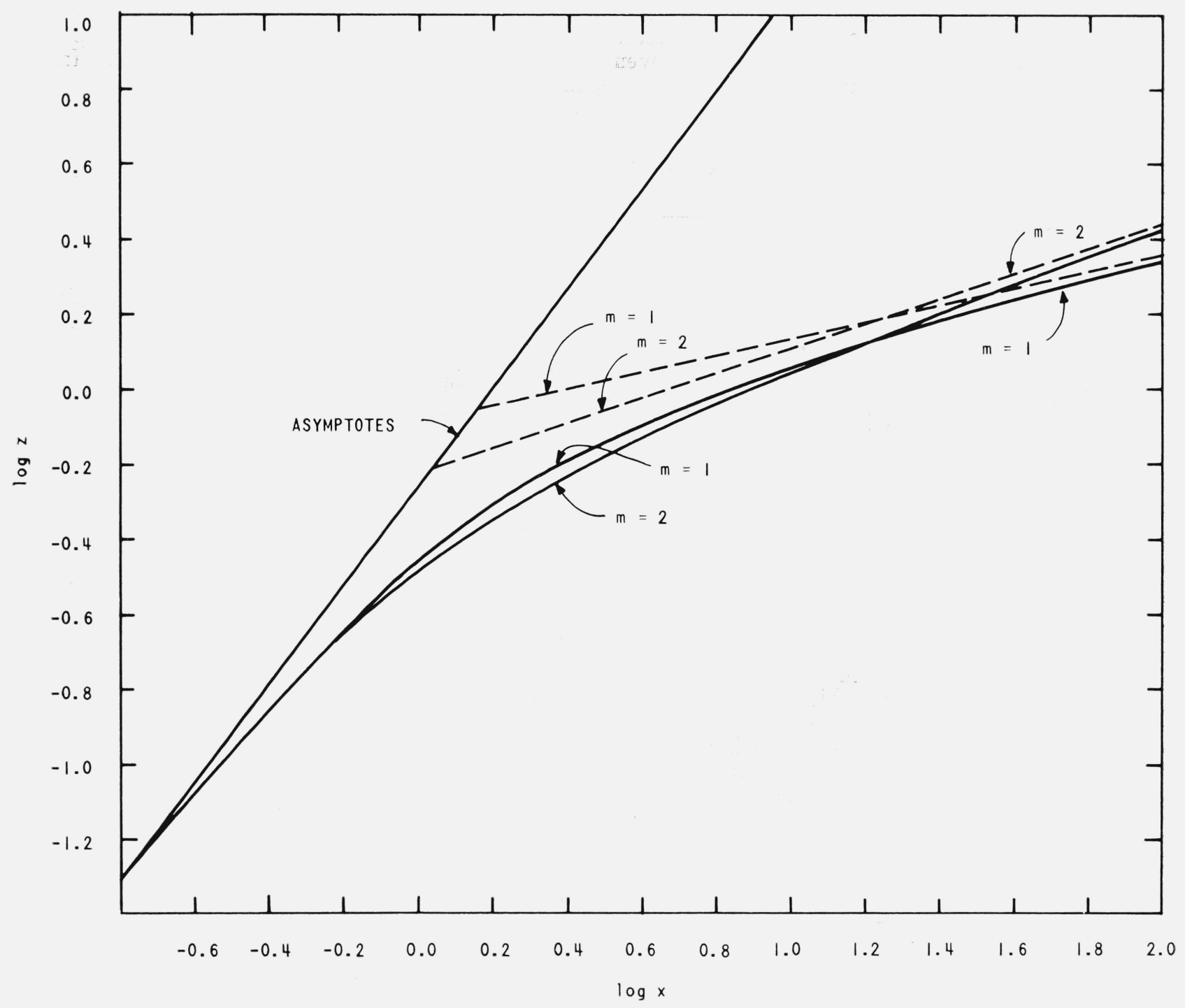

FIGURE 3. Variation of normalized echo duration $\mathrm{z}$ versus normalized line density $\mathrm{x}$ in the transition region.

$$
\begin{aligned}
z & =\frac{\left(\frac{9}{16}\right) x^{4 / 3}}{1+\left(\frac{9}{16} \cdot \frac{e^{2 / 3}}{2^{2 / 3}}\right) \cdot x^{10 / 9}} \\
& \equiv \frac{0.5625 x^{4 / 3}}{1+0.6902 x^{10 / 9}}
\end{aligned}
$$

while for $m=2$, within about 17 percent at worst,

$$
\begin{aligned}
z & =\frac{(9 / 16) x^{4 / 3}}{1+(9 / 16) e^{1 / 2} x} \\
& \equiv \frac{0.5625 x^{4 / 3}}{1+0.9274 x} .
\end{aligned}
$$

Four-place precision is given the constants only for convenience.

\section{Wavelength Dependence of Duration}

From (31) it will be seen that duration is proportional to wavelength squared when attachment is neglected. In the attachment region with $x$ and $z$ $>>1$, and assuming $m=1$, duration varies with wavelength to the power $2 / 3$; for $m=2$, duration varies directly with wavelength. Intermediate behavior occurs in the transition region. Measurements of the exponent of the wavelength variation may then be used to estimate $z$ and $x$.

The wavelength dependence is usually described by the exponent $n$ in the relation $t_{H}=C \lambda^{n} ; C$ is a function of all parameters except wavelength. Then $\ln t_{H}=\ln C+n \ln \lambda$, and $n=d\left(\ln t_{H}\right) / d(\ln \lambda)$. From $(24), \quad \ln z=\ln t_{H}-[2 m /(4+m)]$ ln $\lambda+$ constant. Hence

$$
\frac{d(\ln z)}{d(\ln \lambda)}=\frac{d\left(\ln t_{H}\right)}{d(\ln \lambda)}-\frac{2 m}{4+m}
$$


and

$$
n=\frac{2 m}{4+m}+\frac{d(\ln z)}{d(\ln \lambda)}=\frac{2 m}{4+m}+\frac{d(\ln z)}{d(\ln x)} \cdot \frac{d(\ln x)}{d(\ln \lambda)} .
$$

From (25) $\ln x=[6 /(4+m)]$ ln $\lambda+$ constant, so $d(\ln x) / d(\ln \lambda)=6 /(4+m)$ and

$$
n=\frac{2 m}{4+m}+\frac{6}{4+m} \cdot \frac{d(\ln z)}{d(\ln x)} \text {. }
$$

If $m=0$ (height-independent attachment), from (29) $d(\ln z) / d(\ln x)=(4 / 3) /(z+1)$, and from $(39)$

$$
n=2 /(z+1) \quad \text { for } m=0 .
$$

If $m=1$ (two-body attachment), (39) becomes

$$
n=\frac{2}{5}+\frac{6}{5} \frac{d(\ln z)}{d(\ln x)} \quad \text { for } m=1
$$

Exact values of $n$ versus $x$ or $z$ may be found using $d(\log z) / d(\log x)$ as tabulated in table 1 . The resulting values of $n$ are plotted in figure 6 . If $x<\langle 1, n=2$; if $x\rangle>1, n=2 / 3$. The mid-transition value $n=4 / 3$ occurs when $d(\ln z) / d(\ln x)=7 / 9$. An approximate but explicit relation can be found for $n$ versus $x$ by evaluating the derivative of (41) using the bridging formula (37).

$$
n \cong 2-\frac{4}{3} \frac{0.6902 x^{10 / 9}}{1+0.6902 x^{10 / 9}} \quad \text { for } m=1 .
$$

If $m=2$ (three-body attachment) (39) becomes

$$
n=\frac{2}{3}+\frac{d(\ln z)}{d(\ln x)} \quad \text { for } m=2
$$

for which the mid-transition value $n=3 / 2$ occurs if $d(\ln z) / d(\ln x)=5 / 6$. Evaluating the derivative from the bridging formula (38),

$$
n \cong 2-\frac{0.9274 x}{1+0.9274 x} \quad \text { for } m=2 .
$$

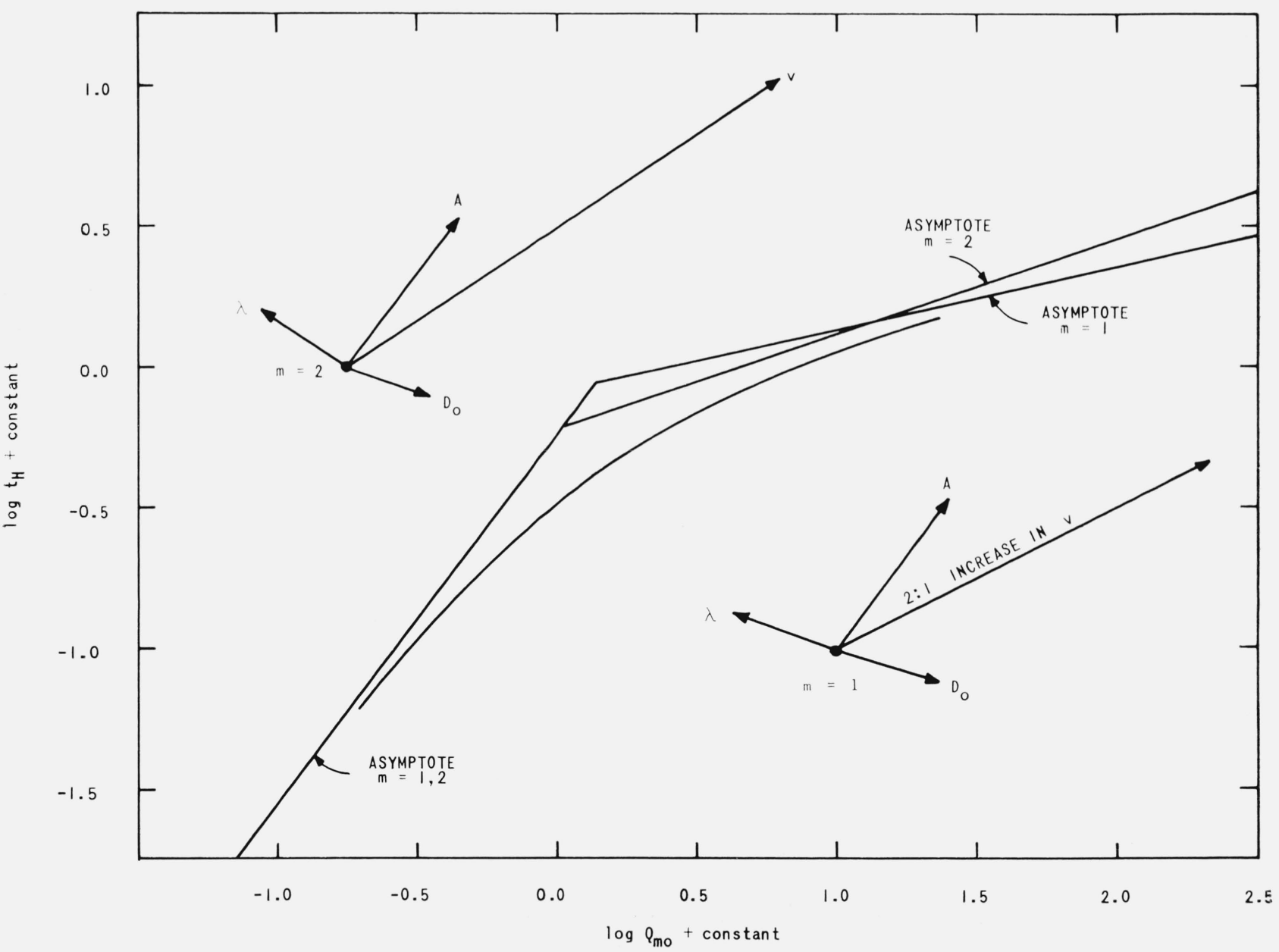

Figure 4. Vectors showing direction and magnitude of shift in $\log \mathrm{t}_{\mathrm{H}}$ versus $\log \mathrm{Q}_{\mathrm{mo}}$ curves for 2 to 1 change in $\mathbf{v}, \lambda$, $\mathrm{A}$, or $\mathrm{D}_{\mathrm{o}}$. 


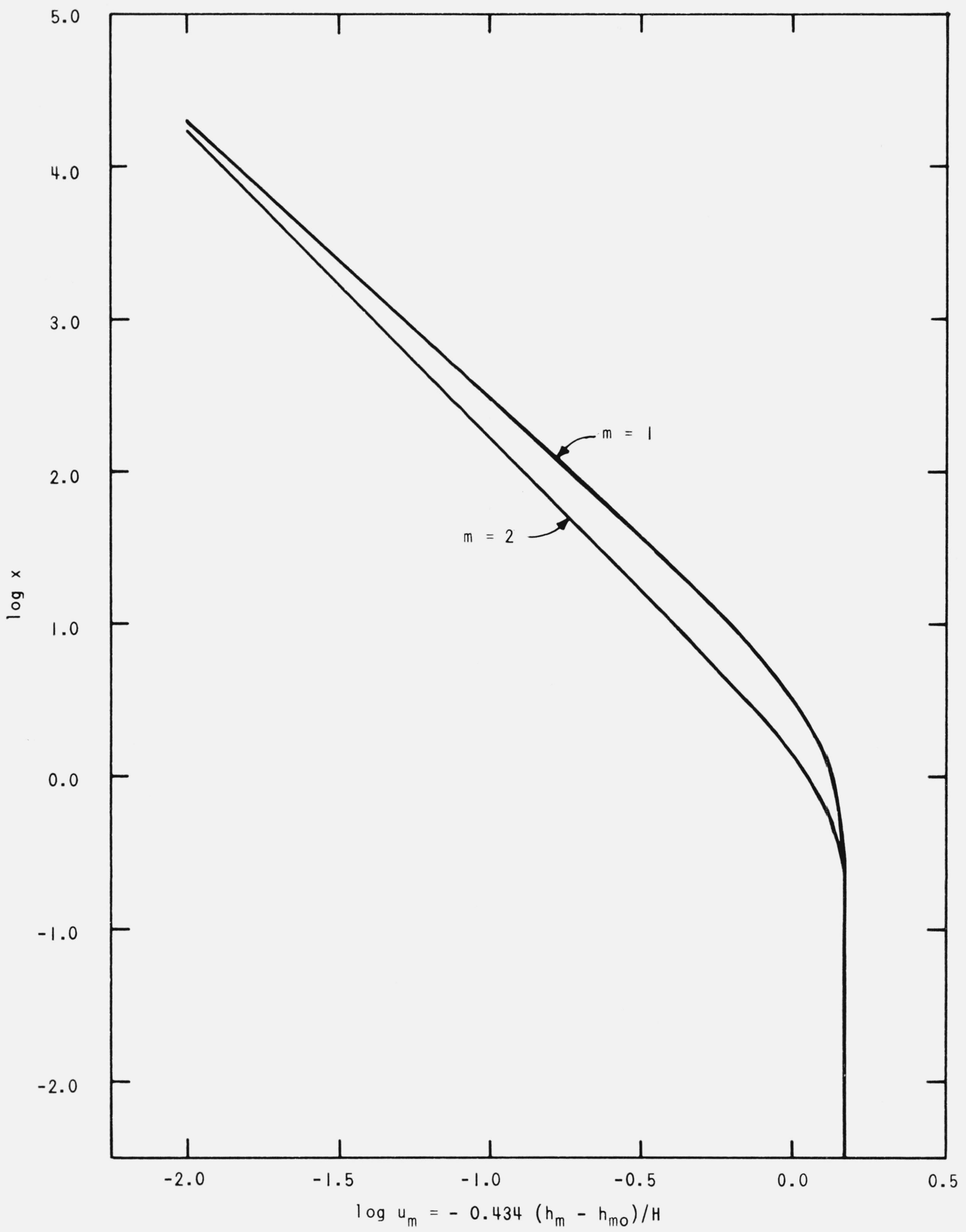

FIGURE 5. The relation between normalized line density $\mathrm{x}$ and $\log \mathrm{u}_{\mathrm{m}}=0.434\left(\mathrm{~h}_{\mathrm{m}}-\mathrm{h}_{\mathrm{mo}}\right) / \mathrm{H} ; \mathrm{h}_{\mathrm{m}}$ is final echoing height, $\mathrm{h}_{\mathrm{m}}$ is height of maximum ionization production. 


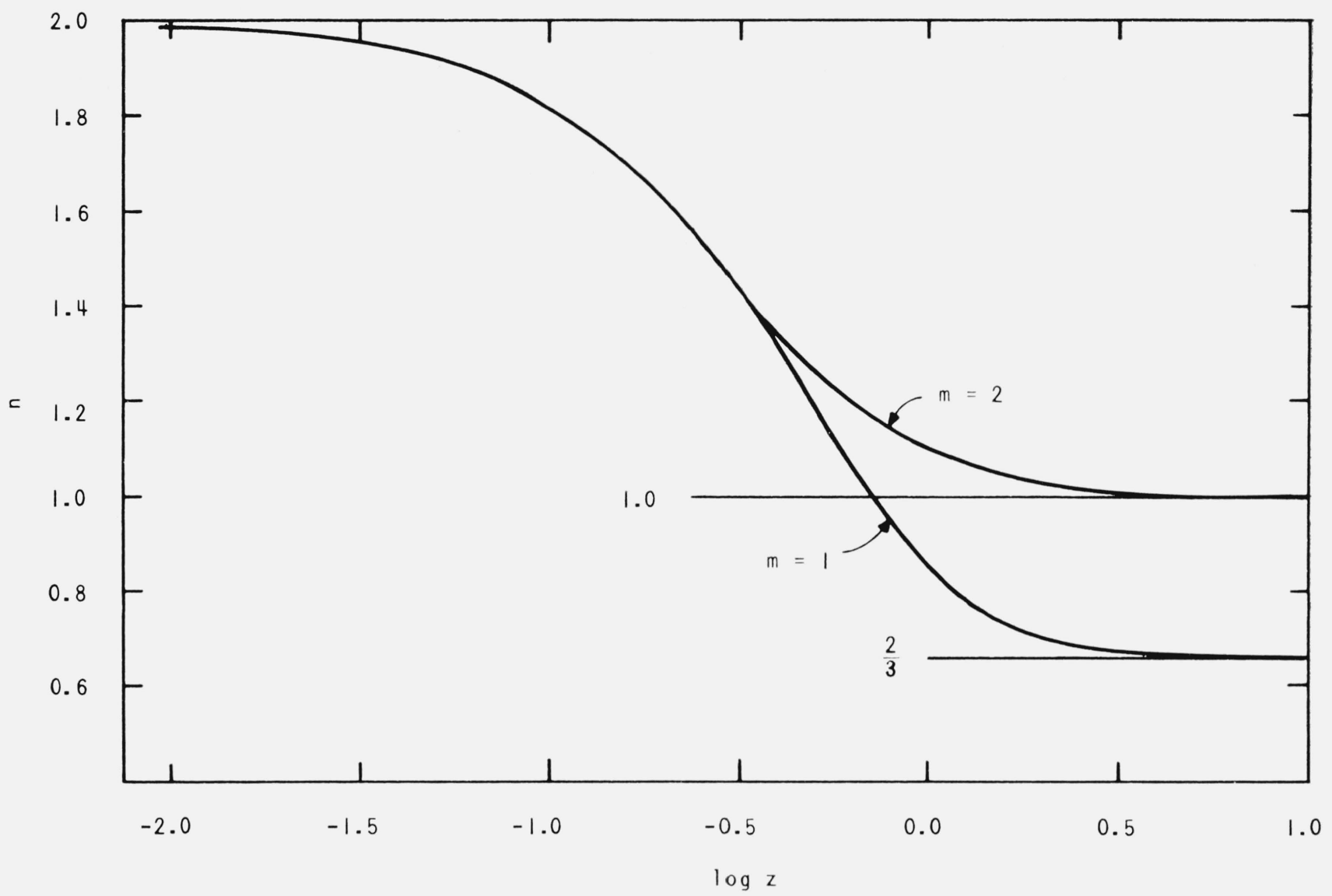

FIGURE 6. Exponent $\mathrm{n}$ of the wavelength dependence of duration, $\mathrm{t}_{\mathrm{H} 2} / \mathrm{t}_{\mathrm{H} 1}=\left(\lambda_{2} / \lambda_{1}\right)^{\mathrm{n}}$, for two- and three-body attachment $(\mathrm{m}=1$ or 2$)$.

\section{Discussion}

The theory of meteor echo duration including attachment, as developed in the present paper, has been kept as general as possible so that detailed comparison can be made with experimental data taken at various laboratories under a variety of conditions. In a companion paper, the experimental results will be analyzed and values deduced for the physical parameters entering into the theory. The valuable earlier work by Davis, Greenhow, and Hall [1959] implies some of the results of the present theory, but unfortunately their curves were obtained largely by numerical or graphical methods applied to particular combinations of wavelength and other parameters, and so are not useful for further development. In addition to being analytic and more general, in some particulars the present results differ from theirs. For instance, Davis, Greenhow, and Hall state [1959, p. 136] that for high line densities the duration is proportional to line density to the power $1 / 3$; the present study shows the exponent to be $2 / 5$ for the two-body attachment process they considered. Moreover, the normalizing factors used by Greenhow and Hall [1962] in plotting their general curves are inconsistent with the present theory; they do not give the derivation leading to these factors.

\section{Conclusions}

In terms of normalized line-density and duration parameters, the duration behavior of overdense meteors can be simply expressed. Most overdense echoes lie in a transition region between a mediumdensity attachment-free domain and a high-density attachment-controlled domain. Values of the normalized parameters may be found from experimental plots of duration versus visual magnitude, wavelength, or meteor velocity; from plots of enduring echo heights versus velocity or magnitude; or from detailed observations of the behavior of individual meteors. From these observations it is possible to deduce the attachment time constant $A$, the type of recombination process $(m)$, the height parameter $K$, the relation between luminous and ionizing efficiency (exponent $J$ ), and the line density corresponding to a given visual magnitude. The diffusion coefficient $D$ and scale height $H$ may be taken from the behavior of underdense trails. Upon inserting the experimental values of these parameters 
(to be derived in a companion paper), the theory can be used to predict echo height and duration as a function of radiofrequency, meteor velocity, radiant position, and line density or magnitude.

\section{References}

Davis, J., J. S. Greenhow, and J. E. Hall (1959), The effect of attachment on radio echo observation of meteors, Proc. Roy. Soc. A 253, 130-139.

Greenhow, J. S., and J. E. Hall (1962), Attachment processes in meteor trails, J. Atmospheric Terrest. Phys. 21, 261-271.
Manning, L. A., and V. R. Eshleman (1959), Meteors in the ionosphere, Proc. IRE 47, 186-199.

McKinley, D. W. R. (1961), Meteor Science and Engineering (McGraw-Hill Book Co., Inc., New York, N.Y.).

Millman, P. M., and D. W. R. McKinley (1956), Meteor echo durations and visual magnitudes, Can. J. Phys. 34, 50-61.

Whipple, F. L. (1955), The physical theory of meteors. VII. On meteor luminosity and ionization, Astrophys. J. 121, 241-249.

(Paper 68D10-407) 